\title{
Clinical significance of Epstein-Barr virus polymerase chain reaction in cerebrospinal fluid
}

\author{
Yong Woo Shin ${ }^{1,2}$, Jun-Sang Sunwoo ${ }^{2,3}$, Han-Sang Lee ${ }^{1,2}$, Woo-Jin Lee ${ }^{1,2}$, Seon-Jae Ahn ${ }^{1,2}$, Sang Kun Lee ${ }^{1}$, Kon Chu ${ }^{1,2}$ \\ ${ }^{1}$ Department of Neurology, Comprehensive Epilepsy Center, Seoul National University Hospital, Seoul National University College of \\ Medicine, Seoul, Korea \\ ${ }^{2}$ Laboratory for Neurotherapeutics, Center for Medical Innovations, Biomedical Research Institute, Seoul National University Hospital, \\ Seoul, Korea \\ ${ }^{3}$ Department of Neurosurgery, Center for Hospital Medicine, Seoul National University Hospital, Seoul, Korea
}

\section{Purpose}

Epstein-Barr virus (EBV) is implicated in various neurological conditions. However, the relationship between EBV DNA in cerebrospinal fluid (CSF) and central nervous system (CNS) infection is unclear. We evaluated the clinical manifestation of patients with EBV DNA detected in CSF.

\section{Methods}

We reviewed the medical records of patients admitted to Seoul National University Hospital from January 2000 to March 2021 who underwent EBV polymerase chain reaction (PCR) tests in CSF. The subjects were divided into positive and negative groups depending on the presence of EBV DNA, and further clinical information was obtained from positive patients.

\section{Results}

CSF EBV PCR tests were performed in 807 patients, and 57 (7.1\%) tested positive. Pleocytosis was common (81.1\%) in CSF samples with EBV DNA detected, and the proportion was significantly higher than that in samples that were EBV PCR negative $(44.5 \%, p<0.0001)$. Among 57 patients with EBV DNA detected in CSF, 51 (89.5\%) were diagnosed with CNS infection or inflammatory disorders. Of the 51 patients, 31 (60.8\%) had possible etiologies other than EBV. Follow-up evaluation was conducted in 19 of 20 patients, and 63.2\% showed a favorable outcome.

\section{Conclusion}

Positive EBV PCR in CSF is mostly nonspecific and should be interpreted with caution. A comprehensive workup is needed to identify other etiologies before considering EBV as the sole culprit.

Keywords: Human herpesvirus 4, Cerebrospinal fluid, Central nervous system infections, Epstein-Barr virus infections

\section{Introduction}

The Epstein-Barr virus (EBV) is a double-stranded DNA virus and a member of the herpes virus family. EBV is a widespread infectious agent worldwide that causes infectious mononucleosis and has a prevalence rate of $90 \%$ [1]. After the first identification of EBV in Burkitt lymphoma in 1964 [2], the association between EBV and other lymphoid malignancies, including primary central nervous system (CNS) lymphoma and epithelial malignancies, has been confirmed $[3,4]$.

EBV infection usually begins in the oral mucosa. Infection re-

Received: August 27, 2021 Revised: October 7, 2021 Accepted: October 13, 2021

Correspondence: Kon Chu

Department of Neurology, Seoul National University Hospital, 101 Daehak-ro, Jongno-gu, Seoul 03080, Korea

E-mail: stemcell.snu@gmail.com

ORCID: https://orcid.org/0000-0001-5863-0302

Copyright (C) 2022 by The Korean Encephalitis and Neuroinflammation Society

This is an open access article distributed under the terms of the Creative Commons Attribution Non-Commercial License (http://creativecommons.org/licenses/by-nc/4.0/) which permits unrestricted non-commercial use, distribution, and reproduction in any medium, provided the original work is properly cited. 
sults from direct fusion of the EBV envelope and the epithelial cell membrane, and the virus proliferates through lytic replication in epithelial cells. After replication in the epithelia, the virus enters B-cells by endocytosis and fuses with the endosome to enter the cytoplasm. In most cases, EBV is asymptomatic in the early stages of infection. However, EBV can later become active and cause infectious diseases such as infectious mononucleosis [5].

EBV has been reported in various neurological conditions, such as encephalitis, meningitis, acute disseminated encephalomyelitis, transverse myelitis, radiculopathy, and cranial nerve palsies $[6,7]$. However, most studies are biased toward patients with lymphoma or infectious mononucleosis, and whether EBV is related to the disease or exists as a bystander is unclear. There are also a few EBV-associated encephalitis case reports, but little is known about the role of EBV in CNS infection. Therefore, we investigated the clinical features of patients with EBV DNA in cerebrospinal fluid (CSF) and possible causes of infection.

\section{Methods}

\section{Study design and population}

We retrospectively analyzed patients admitted to Seoul National University Hospital from January 2000 to March 2021 who underwent CSF analysis, including EBV polymerase chain reaction (PCR). We excluded subjects aged $<18$ years. Patients were also excluded if either CSF cell count was not measured or EBV PCR was performed more than three days after the CSF sample was obtained. We identified 807 adult patients who met the criteria. Based on the EBV PCR results, we categorized the patients into positive and negative groups, and additional clinical information was pursued for the positive patients. Patients who had never tested positive for EBV PCR were labeled as negative patients, and the other cases were labeled positive. As a result, we identified 750 negative patients and 57 positive patients. This study was approved by the Institutional Review Board of Seoul National University Hospital (No. 2009-040-1155) with a waiver of consent because of its retrospective nature.

\section{Data collection}

Demographic and clinical characteristics were collected from electric medical records. If a patient was admitted multiple times, only the initial admission data were selected to obtain demographic information and EBV serology results. For patients with positive CSF EBV PCR, the hospitalization period in which positive samples were obtained for the first time was regarded as the first admission.

All available CSF profile data, including initial and follow-up CSF evaluation, were used for analysis. A CSF specimen was regarded as blood-contaminated if one or more red blood cell (RBC) per $\mathrm{mm}^{3}$ was present in the CSF sample. White blood cell (WBC) count was corrected using blood WBC and RBC count ratio for these samples. We labeled a CSF sample as pleocytosis if the corrected WBC count was greater than five cells $/ \mathrm{mm}^{3}$.

We performed magnetic resonance imaging (MRI) analysis on the EBV-positive patients. The analysis was performed primarily on lesion location, and the lesions were divided into the cortex, subcortical white matter, deep gray matter, brainstem, leptomeninges, and temporal lobe. Temporal lobe lesions were recorded separately. Mass lesions such as tumor or abscess and stroke lesions were excluded from the analysis.

Clinical outcomes were measured using the modified Rankin Scale (mRS) at first admission, first discharge, and last visit. Only patients without another etiology were analyzed to minimize the effect of other diseases on the clinical course.

\section{Statistical analysis}

All statistical analyses were performed using Python (version 3.7.11; Python Software Foundation, Wilmington, DE, USA) and the open-source libraries of SciPy (version 1.4.1; https:// www.scipy.org/). Continuous data are denoted as the median (lower range value-upper range value), and categorical data are expressed as count (percentage). The chi-square or Fisher exact test was used for categorical data, and the Mann-Whitney U-test was used to compare continuous data. The p-values less than 0.05 were considered significant.

\section{Results}

\section{Demographics and serologic profiles}

Among the 807 patients analyzed, 57 (7.1\%) tested positive for CSF EBV PCR (Table 1). The patient group with positive CSF EBV PCR had more male patients $(70.2 \%$ vs. $53.3 \%, \mathrm{p}=0.02)$ and a lower body mass index (BMI) than the negative patient group ( $22 \mathrm{~kg} / \mathrm{m}^{2}$ vs. $23 \mathrm{~kg} / \mathrm{m}^{2}, \mathrm{p}=0.04$ ). There was no difference in age between the two groups. In the total patient group, males had a higher seroprevalence of EBV than females, although the difference was not statistically significant (202 vs. 133, $\mathrm{p}=0.11$ ). Males had lower median BMI than females in the study population ( $23 \mathrm{~kg} / \mathrm{m}^{2}$ vs. $22 \mathrm{~kg} / \mathrm{m}^{2}$, p = 0.02). 
EBV viral capsid antigen (VCA) immunoglobulin (Ig) M antibodies, which suggest acute inflammation [8], were found in $1.6 \%$ of patients and demonstrated no significant difference between the positive and negative groups. EBV VCA IgG was positive in 335 patients (89.6\%), and there was no difference in frequency between the two groups (Table 1).

\section{Cerebrospinal fluid profile}

From the 807 patients, 1,005 CSF samples were collected. EBV PCR was positive in 74 of 1,005 CSF samples (7.4\%), and 931 of 1,005 CSF samples (92.6\%) were negative for EBV PCR. Pleocytosis was common (81.1\%) in CSF samples with EBV PCR positivity, and the proportion was significantly higher than that of the samples with EBV PCR negativity $(44.5 \%$, $\mathrm{p}<0.0001)$. Protein level was higher and glucose level was lower in the EBV PCR positive group (Table 2). The blood contamination ratio was high in the positive group (78.4\% for the positive vs. $59.3 \%$ for the negative, $\mathrm{p}=0.0019$ ). However, only 72 of the contaminated samples $(11.8 \%)$ showed RBC of

Table 1 Demographics and serologic information

\begin{tabular}{|c|c|c|c|}
\hline \multirow{2}{*}{ Variable } & \multicolumn{2}{|c|}{ CSF EBV PCR } & \multirow{2}{*}{ p-value } \\
\hline & Positive $(n=57)$ & Negative $(n=750)$ & \\
\hline \multicolumn{4}{|l|}{ Demographics } \\
\hline Male & $40(70.2)$ & 400 (53.3) & 0.02 \\
\hline Age (yr) & $53(35-65)$ & $52(35-64)$ & 0.42 \\
\hline $\mathrm{BMI}\left(\mathrm{kg} / \mathrm{m}^{2}\right)$ & $22(20-25)$ & $23(21-25)$ & 0.04 \\
\hline \multicolumn{4}{|l|}{ Serology } \\
\hline EBV VCA IgM & 2/41 (4.9) & 5/395 (1.3) & 0.13 \\
\hline EBV VCA IgG & $30 / 31$ (96.8) & 305/343 (88.9) & 0.23 \\
\hline
\end{tabular}

Values are presented as median (range) or count (\%).

CSF, cerebrospinal fluid; EBV, Epstein-Barr virus; PCR, polymerase chain reaction; $\mathrm{BMI}$, body mass index; VCA, viral capsid antigen; Ig, immunoglobulin.
$>1,000 / \mathrm{mm}^{3}$, and there was no significant difference in frequency of samples with $\mathrm{RBC}$ of $>1,000 / \mathrm{mm}^{3}$ between the positive and negative groups ( $8.1 \%$ for the positive vs. $7.1 \%$ for the negative, $\mathrm{p}=0.93$ ).

\section{Diagnosis in the positive group}

If a patient underwent multiple admissions, patient diagnosis was classified based on their disorder on the initial admission. Of the 807 patients, 57 (7.1\%) were positive on EBV PCR (Figure 1). Of the 57 patients who tested positive for EBV PCR, 51 (89.5\%) were diagnosed with CNS infection or inflammatory disorder such as encephalitis, meningitis, ventriculitis, or pachymeningitis (Table 3). The most frequent diagnosis was encephalitis, followed by meningitis and myelitis. The other six patients were diagnosed with malignancy or stroke (Table 3).

Among the 51 patients with CNS infection or inflammatory disorders, 24 (47.1\%) had another etiology, such as herpes simplex virus or $N$-methyl D-aspartate receptor encephalitis, which was identified in the CSF (Table 4). Seven patients

Table 2 Cerebrospinal fluid (CSF) profiles

\begin{tabular}{|c|c|c|c|}
\hline \multirow{2}{*}{ Variable } & \multicolumn{2}{|c|}{ CSF EBV PCR } & \multirow{2}{*}{ p-value } \\
\hline & Positive $(n=74)$ & Negative $(n=931)$ & \\
\hline $\operatorname{RBC}\left(/ \mathrm{mm}^{3}\right)$ & $18(1-158)$ & $2(0-43)$ & 0.0004 \\
\hline WBC $\left(/ \mathrm{mm}^{3}\right)$ & $63(17-228)$ & $4(0-37)$ & $<0.0001$ \\
\hline WBC, corrected $\left(/ \mathrm{mm}^{3}\right)$ & $61(15-228)$ & $4(0-34)$ & $<0.0001$ \\
\hline Glucose $(\mathrm{mg} / \mathrm{dL})$ & $57(46-70)$ & $65(55-75)$ & 0.0005 \\
\hline Protein $(\mathrm{mg} / \mathrm{dL})$ & $91(57-161)$ & $57(41-87)$ & $<0.0001$ \\
\hline Blood contamination (\%) & $58(78.4)$ & $552(59.3)$ & 0.0019 \\
\hline Pleocytosis (\%) & $60(81.1)$ & $414(44.5)$ & $<0.0001$ \\
\hline
\end{tabular}

Values are presented as median (range) or count (\%).

EBV, Epstein-Barr virus; PCR, polymerase chain reaction; RBC, red blood cell; WBC, white blood cell.

Figure 1 Flow chart of the study population

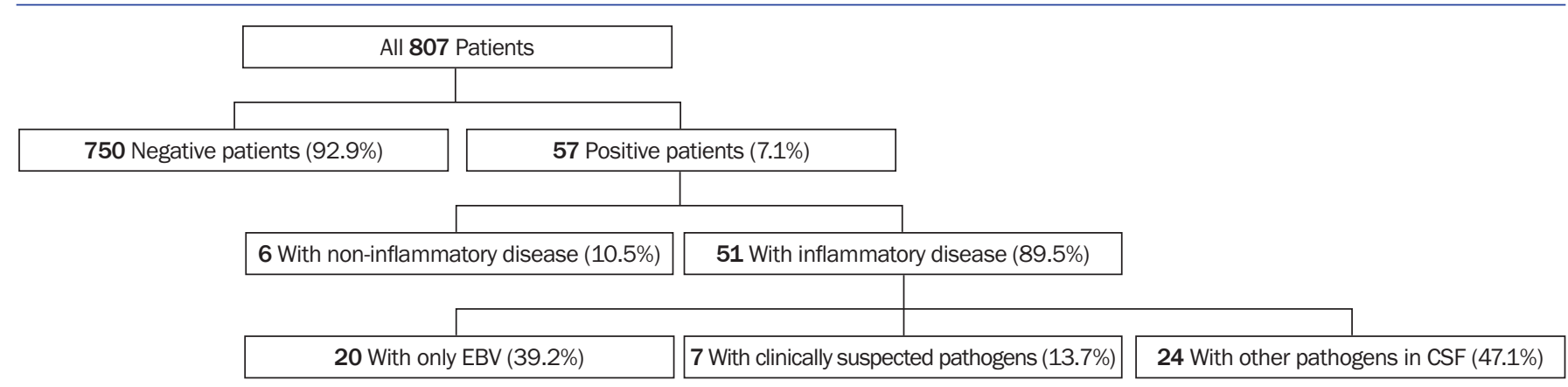

Flow chart of study population selection.

EBV, Epstein-Barr virus; CSF, cerebrospinal fluid. 
Table 3 Diagnoses in cerebrospinal fluid Epstein-Barr virus polymerase chain reaction-positive patients

\begin{tabular}{|c|c|c|}
\hline Category & Diagnosis & Count $(n=57)$ \\
\hline \multirow[t]{13}{*}{ Infection/inflammation (89.5\%) } & Encephalitis & 25 \\
\hline & Meningitis & 10 \\
\hline & Myelitis & 3 \\
\hline & Osteomyelitis & 2 \\
\hline & Brain abscess & 2 \\
\hline & Viral meningitis, with cauda equina syndrome & 1 \\
\hline & Meningoencephalitis with ventriculitis & 1 \\
\hline & Encephalitis with radiculitis & 1 \\
\hline & Cerebral toxoplasmosis & 1 \\
\hline & GBS with irAE & 1 \\
\hline & Encephalomyelitis & 1 \\
\hline & PML & 1 \\
\hline & Ventriculitis & 1 \\
\hline \multirow[t]{4}{*}{ Malignancy (8.8\%) } & PCNSL & $2(3.5)$ \\
\hline & Brain metastasis, ovarian cancer & $1(1.8)$ \\
\hline & Leptomeningeal carcinomatosis & $1(1.8)$ \\
\hline & Intravascular lymphoma & $1(1.8)$ \\
\hline Stroke $(1.8 \%)$ & Cerebral venous sinus thrombosis & $1(1.8)$ \\
\hline
\end{tabular}

Values are presented as count only or count (\%).

GBS, Guillain-Barre syndrome; irAE, immune-related autoimmune encephalitis; PML, progressive multifocal leukoencephalopathy; PCNSL, primary central nervous system lymphoma.

(13.7\%) did not show direct evidence of another etiology in CSF analysis, but other causes were suspected from clinical manifestation, imaging findings, or test results from other samples (Table 4). For example, a recurrent meningitis patient underwent a brain biopsy, which revealed traces of parasitic eggs.

Only 20 of 51 patients (39.2\%) had no possible etiology other than EBV. Among these patients, serum EBV VCA IgM antibodies were detected in only one patient who was diagnosed with encephalitis.

\section{Radiographic findings in the inflammatory group} Among the 51 patients with inflammatory disease, 50 underwent brain MRI. Computed tomography (CT) alone was performed in the other patient. Normal MRI findings were observed for 13 of 50 patients. Leptomeninges was the most frequently involved site, followed by white matter, temporal lobe, and deep gray matter. There was no significant difference between the patients with only EBV PCR positive and the others (Table 5).

In patients with only EBV identified in CSF, 11 were diagnosed with encephalitis. Leptomeningeal enhancement was the most common finding, and cortex and deep gray matter were the next most frequently involved areas (Table 5 and Figure 2). Two patients were diagnosed with myelitis. Both underwent spine MRI, which revealed subtle abnormality on post-con- trast T1-weighted imaging (Figure 2). Among four patients with meningitis, three had normal brain MRI. The other patient did not undergo brain MRI. Instead, a brain CT scan was performed and revealed no abnormality.

\section{Outcomes in Epstein-Barr virus-only patients}

Among the 20 patients with EBV DNA detected in the CSF without another possible etiology (EBV-only patients, hereafter), 19 (95.0\%) were eligible for follow-up evaluation. The outcome was not available for one patient who was transferred to another hospital shortly after emergency room evaluation. Six patients were lost to follow-up after discharge, and the mRS at discharge was regarded as the final mRS.

All 19 patients received antiviral agents. Except for two patients who died of underlying malignancies (bladder cancer and hemophagocytic lymphohistiocytosis), the overall outcome was favorable, with 12 patients $(63.2 \%)$ showing an mRS score of three or less at the last follow-up (Figure 3).

\section{Discussion}

EBV is a highly prevalent virus and has been detected in CSF in various clinical conditions. However, the clinical usefulness of EBV PCR in the context of neuroinflammatory disease has not been determined. In this retrospective study, we evaluated the clinical significance of EBV PCR in CSF and detection 
Table 4 Etiologies of cerebrospinal fluid (CSF) EBV PCR positive patients with inflammatory disorders

\begin{tabular}{|c|c|c|}
\hline Etiology & Count $(n=51)$ & Diagnosis \\
\hline Only EBV (39.2\%) & 20 & Encephalitis (11), meningitis (4), myelitis (2), encephalomyelitis, encephalitis with radiculitis, GBS with irAE ${ }^{\text {a) }}$ \\
\hline \multicolumn{3}{|l|}{ Clinically suspected (13.7\%) } \\
\hline Pseudomonas, Escherichia coli & 1 & Brain abscess \\
\hline $\mathrm{JC}$ virus & 1 & $\mathrm{PML}^{\mathrm{b})}$ \\
\hline $\mathrm{Tb}$ & 2 & Tb meningitis ${ }^{c}$ \\
\hline VZV & 1 & Zoster myelitis \\
\hline MOG & 1 & MOG encephalitis \\
\hline Parasite & 1 & Recurrent meningitis \\
\hline \multicolumn{3}{|l|}{ Directly identified in CSF (47.1\%) } \\
\hline Aspergillus, CMV & 2 & Osteomyelitis (2) \\
\hline CMV & 1 & Encephalitis \\
\hline Enterovirus & 1 & Meningitis \\
\hline $\mathrm{HHV} 8 / 6$ & 1 & Pachymeningitis \\
\hline HSV1 & 2 & Encephalitis (2) \\
\hline HSV1, VZV & 2 & Disseminated herpes zoster infection with encephalitis, disseminated VZV infection with encephalitis \\
\hline HSV2 & 4 & Meningoencephalitis with ventriculitis, encephalitis (2), meningitis with cauda equina syndrome, \\
\hline JBE & 1 & Japanese B encephalitis \\
\hline NMDA receptor $\mathrm{Ab}$ & 3 & NMDARE \\
\hline Pseudomonas aeruginosa & 2 & Ventriculitis (2) \\
\hline RSV B & 2 & Encephalitis (2) \\
\hline Streptococcus & 1 & Brain abscess \\
\hline Toxoplasma & 1 & Toxoplasmosis \\
\hline VDRL reactive & 1 & Probable autoimmune encephalitis ${ }^{d)}$ \\
\hline
\end{tabular}

Values are presented as count.

EBV, Epstein-Barr virus; PCR, polymerase chain reaction; GBS, Guillain-Barre syndrome; irAE, immune-related autoimmune encephalitis; JC virus, John Cunningham virus; PML, progressive multifocal leukoencephalopathy; Tb, tuberculous; VZV, varicella-zoster virus; MOG, myelin oligodendrocyte glycoprotein; CMV, cytomegalovirus; HHV, human herpes virus; HSV, herpes simplex viruses; JBE, Japanese B encephalitis; NMDA, N-methyl-D-aspartic acid; NMDARE, N-methyl D-aspartate receptor encephalitis; RSV, respiratory syncytial virus; VDRL, venereal disease research laboratory.

${ }^{\text {a) } T w o ~ w e e k s ~ a f t e r ~ t h e ~ p a t i e n t ~ r e c e i v e d ~ a t e z o l i z u m a b ~ f o r ~ b l a d d e r ~ c a n c e r, ~ s h e ~ d e v e l o p e d ~ n o n s p e c i f i c ~ i n t e r s t i t i a l ~ p n e u m o n i a ~ a n d ~ h e p a t i t i s, ~ w h i c h ~ l e d ~ t o ~ a d m i s s i o n . ~ S u b-~}$ sequently, she showed altered mentality, dysarthria, and limb weakness. Brain magnetic resonance imaging (MRI) revealed leptomeningeal enhancement, and nerve conduction study demonstrated demyelinating type sensorimotor polyneuropathy. The patient was treated with immunotherapy for irAE and GBS and discharged in a

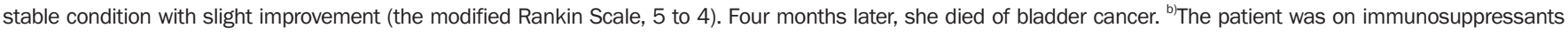
after kidney transplantation when he developed bilateral facial palsy. The CSF revealed pleocytosis, and MRI demonstrated T2 high signal intensity in the left temporal lobe. Initially, the patient received valaciclovir, which was discontinued because of pancytopenia and lack of improvement. Radiologically, PML or central nervous system lymphoma was suspected. Since the brain fluorodeoxyglucose-positron emission tomograph revealed no hypermetabolism, mefloquine was tried. The patient's symptoms improved, and the MRI showed improvement. Biopsy was not performed. ${ }^{\mathrm{c}} \mathrm{A}$ CSF sample showed lymphocytic pleocytosis, the glucose level was low (20 mg/dL), and

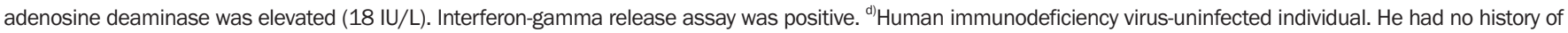
syphilis, and serum rapid plasma regain and fluorescent treponemal antibody absorption test were non-reactive. Hence, the possibility of neurosyphilis was thought to be low. He received antiviral treatment and immunotherapy, considering the possibility of autoimmune encephalitis and the presence of the EBV DNA in CSF.

of EBV DNA. We found that 31 of 51 patients (60.8\%) who tested positive for EBV PCR and had neuroinflammatory conditions had other discernible pathogens and showed a wide range of clinical diagnoses, with encephalitis being the most common. Only 20 of 51 patients (39.2\%) had no possible etiology other than EBV. The patients with only EBV detected in CSF had diverse diagnoses.

The frequency of EBV VCA IgM, which is evidence of acute infection [8], was also low at $2 \%$, and there was no difference in frequency in the positive patients compared with the nega- tive patients ( $1 \%$ for positive patients vs. $5 \%$ for negative patients). However, not all patients underwent EBV serologic evaluation, which can lead to selection bias. Additionally, EBV activity in the blood might not reflect CNS infection. The EBV seroprevalence of the study population was high (89.6\%), consistent with previous reports $[9,10]$, and there is a chance that EBV reactivates in the CNS without apparent systemic reaction. These findings indicate that EBV DNA detection in CSF is insufficient to conclude that EBV caused the neuroinflammatory condition. 
Table 5 Magnetic resonance imaging findings of cerebrospinal fluid EBV PCR positive patients with inflammatory disorders

\begin{tabular}{llll}
\hline & With EBV only $(\mathrm{n}=19)$ & With other pathogens $(\mathrm{n}=\mathbf{3 1})$ & All $(\mathrm{n}=\mathbf{5 0})$ \\
\hline $\begin{array}{l}\text { Normal findings } \\
\text { Abnormal }\end{array} \quad 7(36.8)$ & $7(22.6)$ & $14(28.0)$ \\
$\quad$ Leptomeninges & $8(42.1)$ & $13(41.9)$ & $21(42.0)$ \\
$\quad$ Cortex & $4(21.1)$ & $1(3.2)$ & $5(10.0)$ \\
Temporal lobe & $2(10.5)$ & $4(12.9)$ & $6(12.0)$ \\
Deep gray matter & $4(21.1)$ & $2(6.5)$ & $6(12.0)$ \\
Brainstem & $2(10.5)$ & $1(3.2)$ & $3(6.0)$ \\
Subcortical white matter & $3(15.8)$ & $3(9.7)$ & $6(12.0)$ \\
\hline
\end{tabular}

Values are presented as count (percentage).

EBV, Epstein-Barr virus; PCR, polymerase chain reaction.

Figure 2 MRI findings of CSF EBV PCR positive patients with inflammatory disorders
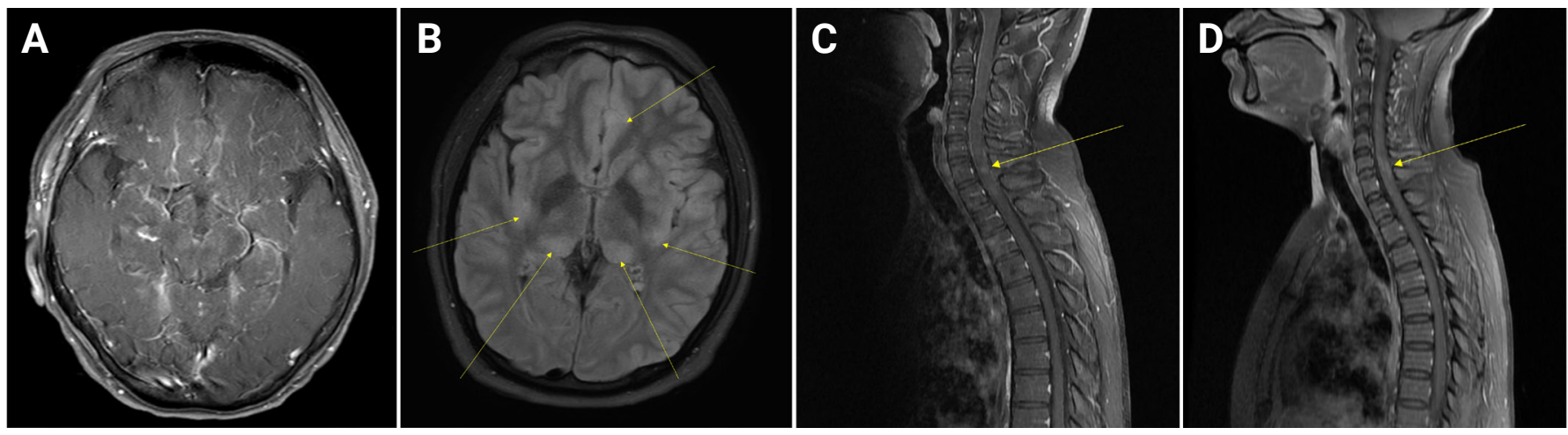

(A) Brain MRI of a 53-year-old woman diagnosed with immune-related autoimmune encephalitis after taking atezolizumab for bladder cancer, showing prominent leptomeningeal enhancement along sylvian fissures, frontal bases, and basal cisterns. (B) Brain MRI of a 31-year-old man, diagnosed with encephalitis, showing relatively symmetric T2 high-SI lesions (yellow arrows) involving bilateral thalamus, basal ganglia, and suspicious both frontal/ temporal cortex. (C) Spine MRI of a 38-year-old man presented with progressive paresthesia of the lower extremities and diagnosed with myelitis, showing subtle multifocal high SI (yellow arrow) at C2-T2 spinal cord on T1CE image. (D) Spine MRI of a 38-year-old woman diagnosed with myelitis, showing suspicious, patchy intramedullary enhancement (yellow arrow) at C5-6.

MRI, magnetic resonance imaging; CSF, cerebrospinal fluid; EBV, Epstein-Barr virus; PCR, polymerase chain reaction; SI, signal intensity; T1CE, T1-weighted contrast-enhanced.

The high proportion of pleocytosis in the CSF EBV PCR positive group suggests that EBV positivity in CSF is associated with neuroinflammatory conditions. However, it does not necessarily mean that EBV caused the neuroinflammation. Instead, EBV DNA might be detected in the CSF because blood-brain barrier (BBB) permeability increases under neuroinflammatory conditions, or EBV in B-cells could have entered the CSF with B-cells during the inflammatory process. Alternatively, since previous studies reported the possibility that EBV could disrupt or injure the BBB [11,12], EBV might predispose patients to other CNS infections. Additionally, the study population consists of heterogeneous groups with various diagnoses. Although we divided the EBV PCR positive patients into groups of neuroinflammatory patients and others, the EBV PCR negative patients were not classified further. Therefore, caution is required in interpretation.

There was a difference in sex ratio and BMI between the positive and negative patients. Although not statistically significant, males had a higher seroprevalence of EBV. In addition, males had a significantly lower BMI. These might partially explain the differences. However, there are limits to interpretation because the two groups consisted of heterogeneous patients.

Although there was a tendency for clinical improvement in EBV-only patients after taking antiviral drugs, whether the effect is from antiviral agents cannot be determined because there was no control group. Considering the insufficient evi- 
Figure 3 Score on modified Rankin Scale

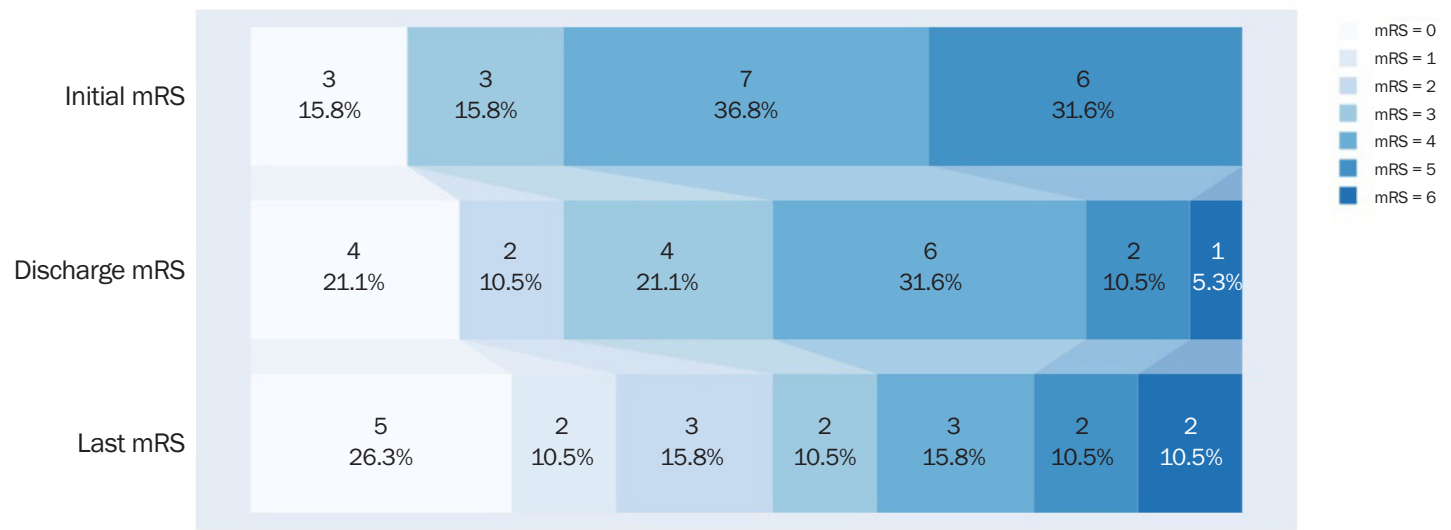

Modified Rankin Scale (mRS) scores at first admission, first discharge, and last visit. Numbers in the different boxes represent the corresponding numbers of patients with that specific mRS.

dence that antiviral drugs are effective in other EBV-associated infections, such as infectious mononucleosis [13], the benefits of antiviral agents in patients with EBV infection in CNS need further investigation.

It is still possible that EBV can cause various neuroinfectious diseases. However, as it does not show a consistent or distinguishing clinical pattern from other neuroinfectious diseases, more detailed research (e.g., brain biopsy) is needed. In addition, no effective antiviral agent is available for EBV. Therefore, the clinical usefulness of EBV PCR in CSF is not significant unless the patient is suspected of having a condition known to be associated with EBV, such as CNS lymphoma or nasopharyngeal tumor.

This study has several limitations. First, this is a retrospective study. We analyzed clinical and laboratory information using only patient medical records. Since there was a lack of standardized evaluation protocol and treatment strategies, patients underwent different workups and treatments according to clinical status. Second, the study population consists of heterogeneous groups with various diagnoses. Although we divided the EBV PCR positive patients into groups of neuroinflammatory patients and others, the EBV PCR negative patients were not classified further. Third, the ratio of blood contamination in EBV-positive patients was high in our data, and the contaminated blood in CSF samples might have caused false-positive results. However, the probability of false positives might be low given that the amount of contaminated RBC was low in most samples. Fourth, the study was conducted with a small number of samples from a single institution.
Therefore, the study population might not represent general EBV-positive patients. However, the tertiary care setting made it possible to conduct comprehensive etiologic evaluation, which revealed that a large proportion of the patients with CSF EBV PCR had other etiologies.

In conclusion, patients positive for CSF EBV PCR commonly showed pleocytosis and were diagnosed with CNS inflammatory disorders. However, the positive patients had diverse clinical aspects, and more than half of the patients had another discernable etiology. Additionally, the frequency of EBV VCA IgM was low at $1 \%$ in EBV PCR-positive patients. Therefore, positive EBV PCR in CSF is nonspecific and does not necessarily indicate that EBV caused neuroinflammation. A comprehensive workup is needed to identify another etiology before considering EBV as the culprit. Considering that no effective antiviral agent is available for EBV, and the possibility of EBV causing neuroinfection is low, the clinical usefulness of EBV PCR in CSF is not significant unless the patient is suspected of having a condition known to be associated with $\mathrm{EBV}$, such as CNS lymphoma or nasopharyngeal tumor.

\section{Conflicts of Interest}

Kon Chu and Sang Kun Lee have been on the editorial board of encephalitis since October 2020. They were not involved in the review process of this article. No other potential conflict of interest relevant to this article was reported. 


\section{Author Contributions}

Conceptualization: Sunwoo JS, Lee SK, Chu K; Data curation: Shin YW; Formal analysis: Shin YW; Methodology: Shin YW, Sunwoo JS, Lee HS, Lee WJ, Ahn SJ, Chu K; Visualization: Shin YW; Supervision: Lee SK, Chu K; Validation: Sunwoo JS, Chu K; Writing-original draft: Shin YW; Writing-review \& editing: Shin YW, Sunwoo JS, Lee HS, Lee WJ, Ahn SJ, Lee SK, Chu K.

\section{References}

1. Niedobitek G, Meru N, Delecluse HJ. Epstein-Barr virus infection and human malignancies. Int J Exp Pathol 2001;82:149-170.

2. Epstein MA, Achong BG, Barr YM. Virus particles in cultured lymphoblasts from Burkitt's lymphoma. Lancet 1964;1:702-703.

3. Ayee R, Ofori ME, Wright E, Quaye O. Epstein Barr virus associated lymphomas and epithelia cancers in humans. J Cancer 2020;11:17371750.

4. Corcoran C, Rebe K, van der Plas H, Myer L, Hardie DR. The predictive value of cerebrospinal fluid Epstein-Barr viral load as a marker of primary central nervous system lymphoma in HIV-infected persons. J Clin Virol 2008;42:433-436.

5. Stanfield BA, Luftig MA. Recent advances in understanding Ep-
stein-Barr virus. F1000Res 2017;6:386.

6. Connelly KP, DeWitt LD. Neurologic complications of infectious mononucleosis. Pediatr Neurol 1994;10:181-184.

7. Fujimoto H, Asaoka K, Imaizumi T, Ayabe M, Shoji H, Kaji M. Epstein-Barr virus infections of the central nervous system. Intern Med 2003;42:33-40.

8. De Paschale M, Clerici P. Serological diagnosis of Epstein-Barr virus infection: problems and solutions. World J Virol 2012;1:31-43.

9. Kang CI, Choi CM, Park JT, Park TS. Seroprevalence of Epstein-Barr virus infection in young men of South Korea. Infect Chemother 2007;39: 93-94.

10. Oh SH, Lee YA, Moon WY, et al. Prevalence of Epstein-Barr virus (EBV) antibody in Korean children. J Korean Pediatr Soc 1994;37:804-811.

11. Indari O, Chandramohanadas R, Jha HC. Epstein-Barr virus infection modulates blood-brain barrier cells and its co-infection with Plasmodium falciparum induces RBC adhesion. Pathog Dis 2021;79:ftaa080.

12. Casiraghi C, Dorovini-Zis K, Horwitz MS. Epstein-Barr virus infection of human brain microvessel endothelial cells: a novel role in multiple sclerosis. J Neuroimmunol 2011;230:173-177.

13. De Paor M, O’Brien K, Fahey T, Smith SM. Antiviral agents for infectious mononucleosis (glandular fever). Cochrane Database Syst Rev 2016;12:CD011487. 